\title{
MICE and NuStorm
}

\author{
M. Bonesini* ${ }^{*}$ \\ Sezione INFN Milano Bicocca, Dipartimento di Fisica G. Occhialini \\ Università di Milano Bicocca, Milano, Italy. \\ E-mail: maurizio.bonesini@mib.infn.it
}

A rich $R \& D$ program to study neutrinos directly produced from high brilliance muon beams is under way. The implementation of the neutrino factory and muon collider concepts require a complete understanding of all parameters involved in ionization cooling. A full study of these has been done within the MICE experimental at RAL, reconstructing emittance evolution with a particle-by-particle method. A shortcut to this problem may be the NuStorm proposal, providing neutrino beams from accelerated muons using only conventional accelerator techniques. First results on the experimental evidence of muon cooling via ionization are shown in this paper.

Neutrino Oscillation Workshop (NOW2018)

9 - 16 September, 2018

Rosa Marina (Ostuni, Brindisi, Italy)

\footnotetext{
* Speaker.

${ }^{\dagger}$ A footnote may follow.
} 


\section{Introduction}

The Muon Collider (MC) and Neutrino Factory (NF) concepts, based on high brilliance muon beams, have been proposed since the early 60's. Their design has been optimized in references [1], [2] and [3]. While a MC looks at the high-energy frontier, precise Higgs physics and beyond, a NF addresses the precision frontier: looking at $\mathrm{CP}$-violation in the neutrino sector. The current design of a NF or a MC front-end is similar, up to the beginning of the cooling section [4] and thus a staged approach may be devised. The most critical issue for the development of a NF or a MC is still the cooling of muons. Muons are produced as tertiary particles in the chain of reactions $p A \mapsto \pi X, \pi \mapsto \mu \nu$ and thus are spread over a large longitudinal and transverse phase space. Present accelerator technologies require input beams with small phase space. To solve this mismatch one may use either new large aperture accelerators, as FFAG machines [5] or try to reduce ("cool”) the incoming muon beam phase space. While for a NF a small cooling factor is needed: $\sim 2.4$ in the IDS-NF design [3], for a MC a much higher cooling factor: $\sim 2 \times 10^{6}$ is required.

\section{The MICE experiment at RAL}

The only effective way to cool muons, due to their short lifetime: $\tau \sim 2.2 \mu \mathrm{s}$, is the so-called "ionization cooling". It is accomplished by passing muons through a low-Z absorber, where they loose energy by ionization and only the longitudinal component of momentum is replenished by RF cavities [6]. The MICE experiment [7] had as initial goal to study a fully engineered cooling cell of US Study 2 [2], see Figure 1 for further details. This aim has been downsized in 2014 to a demonstration of ionization cooling with a simplified lattice (see Figure 3).
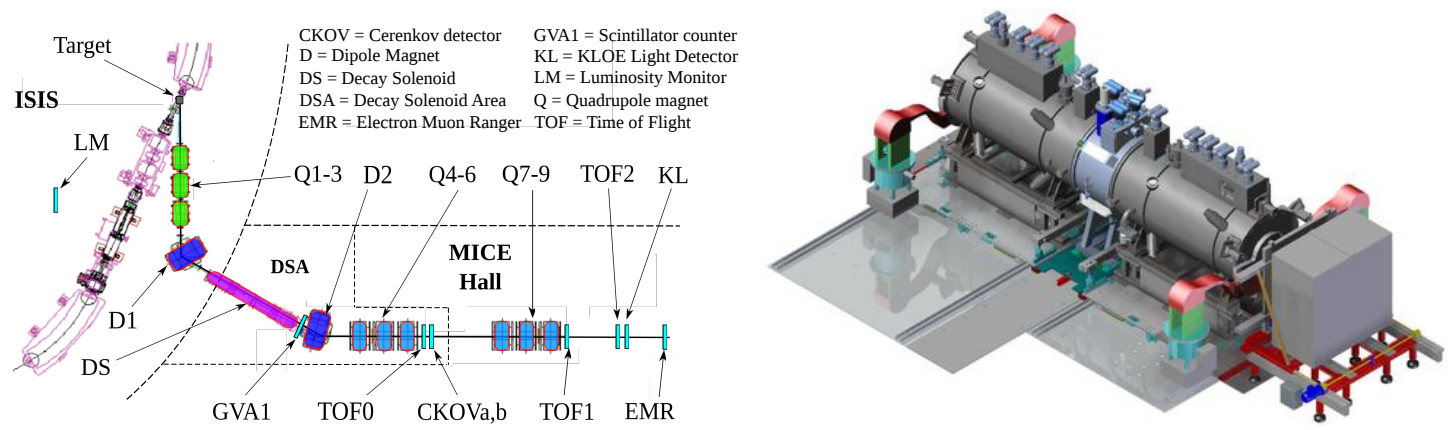

Figure 1: Left panel: schematic layout of the instrumented MICE beamline at RAL. Right panel: layout of the MICE cooling channel during STEP IV, dedicated to the study of factors affecting ionization cooling.

A dedicated muon beam from ISIS (140-240 MeV/c central momentum, with tunable input emittance in the range $3-10 \pi \cdot \mathrm{mm}$ rad) enters the MICE cooling section after a lead diffuser of adjustable thickness. The MICE beamline has been characterized in summer 2010 [8], by using the TOF detectors [9] ( $\sim 50$ ps resolution), As conventional emittance measurement techniques reach barely a $10 \%$ precision, the final high-precision measure of emittance has been done in MICE on a particle-by-particle basis by measuring $x, y, x^{\prime}=p_{x} / p_{z}, y^{\prime}=p_{y} / p_{z}, E, t$ with trackers, inside $4 \mathrm{~T}$ superconducting solenoids, and the TOF system. An example of measured transverse emittance is reported in Figure 2. The total uncertainty is between 2 and $4 \%$ as a function of beam momentum. For details see reference [10]. The fact that the number of muons in the beam core 
decreases/increases with no absorber or with a $\mathrm{LiH}, \mathrm{LH}_{2}$ absorber, as shown in Figure 2, is a demonstration of ionization cooling. Small emittance beams have more particles occupying lower amplitudes.
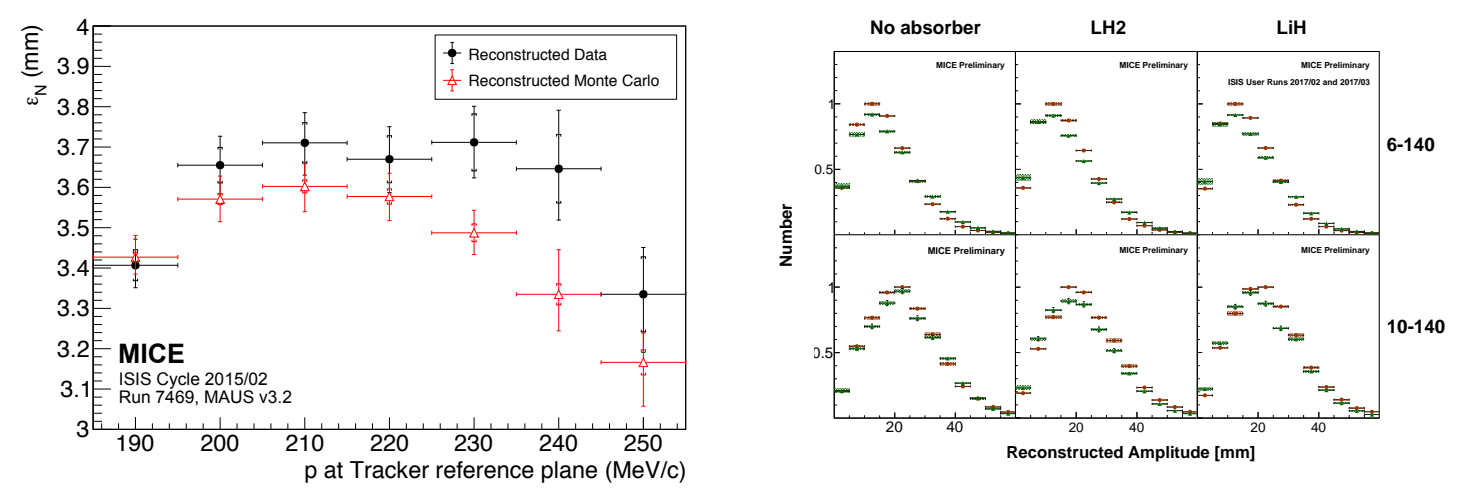

Figure 2: Left panel: normalized transverse emittance $\varepsilon_{N}$ as a function of momentum p. The inner error bars refer to statistical uncertainty, while the outer error bars include systematics. Right panel: number of particles of different amplitude upstream (red) and downstream (green) of the absorbers. Histograms are normalized to the peak bin in the upstream sample.

\section{A view into the next future}

Measurement of a sustainable ionization cooling requires the addition of RF cavities to restore muon energy losses in the absorber materials. The original MICE design was revised to reduce costs and have a streamlined timescale. The simplified setup is presented in Figure 3 [11]. The cooling cell will include now only two available $201 \mathrm{MHz}$ cavities, with $\mathrm{LiH}$ absorbers. The expected performances are shown in the right panel of Figure 3. Unfortunately, budget problems have restrained the MICE collaboration to go beyond STEP IV and proceed to the "demo cooling".

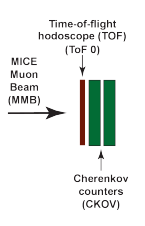

MICE

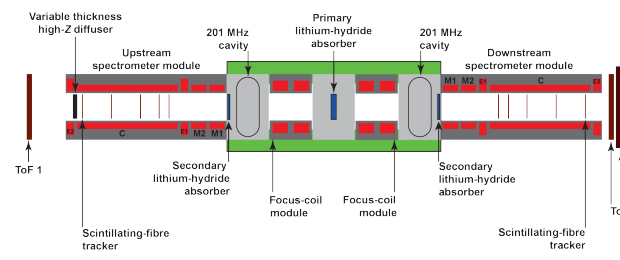

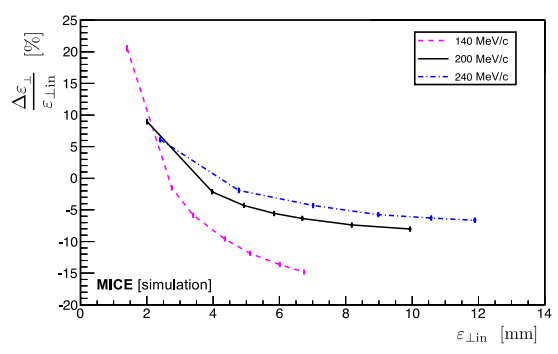

Figure 3: Left panel: a schematic layout of the foreseen "demo cooling" experiment. Right panel: foreseem performances of the "demo cooling" lattice.

As a shortcut to the problem of cooling of muons, NuStorm has been put forward as the simplest implementation of the Neutrino Factory concept in recent years [12]. It is based on conventional accelerator techniques. A racetrack-like muon storage ring is put at the heart of the facility and from the decay of $\mu^{ \pm}$both $\bar{v}_{\mu}$ and $\bar{v}_{e}$ beams may be delivered. A schematic layout is shown 
in Figure 4. Up to the target, where pions are focussed by a magnetic horn, NuStorm is identical to a conventional neutrino beam. After the horn pions are injected into the decay ring, where they decay and produce muons that are trapped inside. Neutrino beams from muon decay have a precise flavour content and energy (the muon energy being defined by the lattice) and their flux may be measured with high precision $(\leq 1 \%)$.

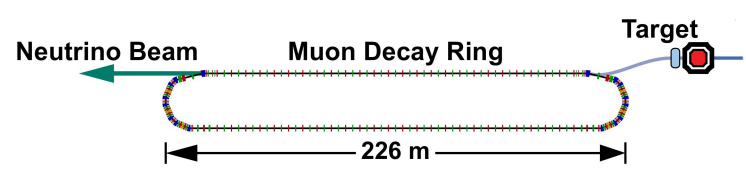

Figure 4: Schematic layout of the foreseen NuSTORM facility.

The physics program includes short baseline oscillation searches for sterile neutrinos, detailed studies of neutrino-nucleus scattering in the region of interest for future LBL neutrino beams and may be a testbed for future accelerator studies. CERN would be an ideal host for this facility [13].

\section{Conclusions}

Muon ionisation cooling is needed for both the NF and the MC projects. It is been experimentally measured by MICE as a function of $\mathrm{p}$ and $\beta_{\perp}$ at the absorber. The NuStorm concept, using only available accelerator techniques, may be a clever shortcut towards a NF, albeit at lower energy.

\section{References}

[1] Geer S., LINAC10 Conference, Tsukuba, Japan, 2010, xarXiv:1202.2140.

[2] S.Ozaki et al., BNL-52623, June 2001 ; M.M. Alsharo'a et al., Phys. Rev. ST. Accel. Beams 6,081001 (2003), arXiv:hep-ex/0207031; R. Palmer et al., arXiv:0711.4275.

[3] Choubey S. et al, Int. Design Study for the Neutrino Factory, IDS-NF-20,2011, arXiv:1112.2853; M. Apollonio et al., CERN-TH-2002-208, 2002;

M. Bogomilov et al., Phys. Rev. ST. Accel.Beams. 17(2014)18,121002.

[4] M. Bonesini, Frascati Phys. Ser. 61 (2016) 11.

[5] Rees, G.H. and Kelliher, D.J., Proceedings 46th ICFA Advanced Beam Dynamics Workshop HB2010,2010, p. 54-56; Edgecock R., Int.J.Mod.Phys.A26 (2011) 1736-1743.

[6] A.N. Skrinsky and V.V. Parkhomchuk, Sov. J. Part. Nucl. 12 (1981) 223.

[7] G. Gregoire et al. [MICE coll.] , MICE Proposal to RAL, 2003.

[8] D. Adams et al. [MICE coll.] Eur. Phys. J. C73 (2013) 2582.

[9] R. Bertoni et al., Nucl. Instr. and Meth. A615 (2010) 14, arXiv:001.4426.

[10] D.Adams et al. [MICE coll.], arXiv:1810.13224, submitted to Eur. Phys. J. C.

[11] M. Bogomilov et al. [MICE coll.], Phys. Rev. Accel. Beams 20 (2017) no. 6, 063501.

[12] K.Long, J.Phys.Conf.Ser. 1056 (2018) no. 1,012033;

D.Adey et al., Ann. ReV. Nucl. Part. Sci. 65 (2015) 145.

[13] NuStorm Coll., Expression of Interest to CERN, arXiv:1305.1419. 